\title{
115.
}

\section{NOTE ON THE PORISM OF THE IN-AND-CIRCUMSCRIBED POLYGON.}

[From the Philosophical Magazine, vol. vi. (1853), pp. 99-102.]

THE equation of a conic passing through the points of intersection of the conics

is of the form

$$
U=0, \quad V=0
$$

$$
w U+V=0,
$$

where $w$ is an arbitrary parameter. Suppose that the conic touches a given line, we have for the determination of $w$ a quadratic equation, the roots of which may be considered as parameters for determining the line in question. Let one of the values of $w$ be considered as equal to a given constant $k$, the line is always a tangent to the conic

$$
k U+V=0
$$

and taking $w=p$ for the other value of $w, p$ is a parameter determining the particular tangent, or, what is the same thing, the point of contact of this tangent.

Suppose the tangent meets the conic $U=0$ (which is of course the conic corresponding to $w=\infty)$ in the points $P, P^{\prime}$, and let $\theta, \infty$ be the parameters of the point $P$, and $\theta^{\prime}, \infty$ the parameters of the point $P^{\prime}$. It follows from my "Note on the Geometrical representation of the Integral $\int d x \div \sqrt{(x+a)(x+b)(x+c)}, "$ [113] $\left(^{1}\right)$ and from the theory of invariants, that if $\square \xi$ represent the "Discriminant" of $\xi U+V$

${ }^{1}$ I take the opportunity of correcting an obvious error in the note in question, viz. $a^{2}+b^{2}+c^{2}-2 b c-2 c a-2 a b$ is throughout written instead of (what the expression should be) $b^{2} c^{2}+c^{2} a^{2}+a^{2} b^{2}-2 a^{2} b c-2 b^{2} c a-2 c^{2} a b$. [This correction is made, ante p. 55.] 
(I now use the term discriminant in the same sense in which determinant is sometimes used, viz. the discriminant of a quadratic function $a x^{2}+b y^{2}+c z^{2}+2 f y z+2 g z x+2 h x y$ or $(a, b, c, f, g, h)(x, y, z)^{2}$, is the determinant $\left.k=a b c-a f^{2}-b g^{2}-c h^{2}+2 f g h\right)$, and if

$$
\Pi \xi=\int_{\infty} \frac{d \xi}{\sqrt{\square \xi}},
$$

then the following theorem is true, viz.

"If $(\theta, \infty),\left(\theta^{\prime}, \infty\right)$ are the parameters of the points $P, P^{\prime}$ in which the conic $U=0$ is intersected by the tangent, the parameter of which is $p$, of the conic $k U+V=0$, then the equations

$$
\begin{aligned}
& \Pi \theta=\Pi p-\Pi k, \\
& \Pi \theta^{\prime}=\Pi p+\Pi k,
\end{aligned}
$$

determine the parameters $\theta, \theta^{\prime}$ of the points in question." And again,-

"If the variable parameters $\theta, \theta^{\prime}$ are connected by the equation

$$
\Pi \theta^{\prime}-\Pi \theta=2 \Pi k \text {, }
$$

then the line $P P^{\prime}$ will be a tangent to the conic $k U+V=0 . "$ Whence, also,-

"If the sides of a triangle inscribed in the conic $U=0$ touch the conics

$$
\begin{aligned}
& k U+V=0, \\
& k^{\prime} U+V=0, \\
& k^{\prime \prime} U+V=0,
\end{aligned}
$$

then the equation

$$
\Pi k+\Pi k^{\prime}+\Pi k^{\prime \prime}=0
$$

must hold good between the parameters $k, k^{\prime}, k^{\prime \prime}$."

And, conversely, when this equation holds good, there are an infinite number of triangles inscribed in the conic $U=0$, and the sides of which touch the three conics; and similarly for a polygon of any number of sides.

The algebraical equivalent of the transcendental equation last written down is

$$
\left|\begin{array}{lll}
1, & k, & \sqrt{\square k} \\
1, & k^{\prime}, & \sqrt{\square k^{\prime}} \\
1, & k^{\prime \prime}, & \sqrt{\square k^{\prime \prime}}
\end{array}\right|=0 ;
$$

let it be required to find what this becomes when $k=k^{\prime}=k^{\prime \prime}=0$, we have

$$
\sqrt{\square k}=A+B k+C k^{2}+\ldots,
$$


and substituting these values, the determinant divides by

$$
\left|\begin{array}{lll}
1, & k, & k^{2} \\
1, & k^{\prime}, & k^{\prime 2} \\
1, & k^{\prime \prime}, & k^{\prime 2}
\end{array}\right|
$$

the quotient being composed of the constant term $C$, and terms multiplied by $k, k^{\prime}, k^{\prime \prime}$; writing, therefore, $k=k^{\prime}=k^{\prime \prime}=0$, we have $C=0$ for the condition that there may be inscribed in the conic $U=0$ an infinity of triangles circumscribed about the conic $V=0 ; C$ is of course the coefficient of $\xi^{2}$ in $\sqrt{\square \xi}$, i.e. in the square root of the discriminant of $\xi U+V$; and since precisely the same reasoning applies to a polygon of any number of sides,-

Theorem. The condition that there may be inscribed in the conic $U=0$ an infinity of $n$-gons circumscribed about the conic $V=0$, is that the coefficient of $\xi^{n-1}$ in the development in ascending powers of $\xi$ of the square root of the discriminant of $\xi U+V$ vanishes. [This and the theorem p. 90 are erroneous, see post, 116].

It is perhaps worth noticing that $n=2$, i. e. the case where the polygon degenerates into two coincident chords, is a case of exception. This is easily explained.

In particular, the condition that there may be in the conic $^{1}$

$$
a x^{2}+b y^{2}+c z^{2}=0
$$

an infinity of $n$-gons circumscribed about the conic

$$
x^{2}+y^{2}+z^{2}=0,
$$

is that the coefficient of $\xi^{n-1}$ in the development in ascending powers of $\xi$ of

$$
\sqrt{(1+a \xi)(1+b \xi)(1+c \xi)}
$$

vanishes; or, developing each factor, the coefficient of $\xi^{n-1}$ in

$$
\left(1+\frac{1}{2} a \xi-\frac{1}{8} a^{2} \xi^{2}+\frac{1}{16} a^{3} \xi^{3}-\frac{5}{64} a^{4} \xi^{4}+\& c .\right)\left(1+\frac{1}{2} b \xi-\& c .\right)\left(1+\frac{1}{2} c \xi-\& c .\right)
$$

vanishes.

Thus, for a triangle this condition is

$$
a^{2}+b^{2}+c^{2}-2 b c-2 c a-2 a b=0 ;
$$

for a quadrangle it is

$$
a^{3}+b^{3}+c^{3}-b c^{2}-b^{2} c-c a^{2}-c^{2} a-a b^{2}-a^{2} b+2 a b c=0,
$$

which may also be written

$$
(b+c-a)(c+a-b)(a+b-c)=0
$$

and similarly for a pentagon, \&c.

${ }^{1}$ I have, in order to present this result in the simplest form, purposely used a notation different from that of the note above referred to, the quantities $a x^{2}+b y^{2}+c z^{2}$ and $x^{2}+y^{2}+z^{2}$ being, in fact, interchanged.

C. II. 
Suppose the conics reduce themselves to circles, or write

$$
\begin{aligned}
& U=x^{2}+y^{2}-R^{2}=0, \\
& V=(x-a)^{2}+y^{2}-r^{2}=0 ;
\end{aligned}
$$

$R$ is of course the radius of the circumscribed circle, $r$ the radius of the inscribed circle, and $a$ the distance between the centres. Then

$$
\xi U+V=\left(\xi+1, \xi+1,-\xi R^{2}-r^{2}+a^{2}, 0,-a, 0\right)(x, y, 1)^{2},
$$

and the discriminant is therefore

$$
-(\xi+1)^{2}\left(\xi R^{2}+r^{2}-a^{2}\right)-(\xi+1) a^{2}=-(1+\xi)\left\{r^{2}+\xi\left(r^{2}+R^{2}-a^{2}\right)+\xi^{2} R^{2}\right\} .
$$

Hence,

Theorem. The condition that there may be inscribed in the circle $x^{2}+y^{2}-R^{2}=0$ an infinity of $n$-gons circumscribed about the circle $(x-a)^{2}+y^{2}-r^{2}=0$, is that the coefficient of $\xi^{n-1}$ in the development in ascending powers of $\xi$ of

may vanish.

$$
\sqrt{(1+\xi)\left\{r^{2}+\xi\left(r^{2}+R^{2}-a^{2}\right)+\xi^{2} R^{2}\right\}}
$$

Now

$$
\left(A+B \xi+C \xi^{2}\right)^{\frac{1}{2}}=\sqrt{A}\left\{1+\frac{1}{2} B \frac{\xi}{A}+\left(\frac{1}{2} A C-\frac{1}{8} B^{2}\right) \frac{\xi^{2}}{A^{2}}+\ldots\right\},
$$

or the quantity to be considered is the coefficient of $\xi^{n-1}$ in

$$
\left(1+\frac{1}{2} \xi-\frac{1}{8} \xi^{2} \cdots\right)\left\{1+\frac{1}{2} B \frac{\xi}{A}+\left(\frac{1}{2} A C-\frac{1}{8} B^{2}\right) \frac{\xi^{2}}{A^{2}}+\ldots\right\},
$$

where, of course,

$$
A=r^{2}, \quad B=r^{2}+R^{2}=a^{2}, \quad C=R^{2} .
$$

In particular, in the case of a triangle we have, equating to zero the coefficient of $\xi^{2}$,

$$
(A-B)^{2}-4 A C=0
$$

or substituting the values of $A, B, C$,

$$
\left(a^{2}-R^{2}\right)^{2}-4 r^{2} R^{2}=0 \text {, }
$$

that is

$$
\left(a^{2}-R^{2}+2 R r\right)\left(a^{2}-R^{2}-2 R r\right)=0 ;
$$

the factor which corresponds to the proper geometrical solution of the question is

$$
a^{2}-R^{2}+2 R r=0,
$$

Euler's well-known relation between the radii of the circles inscribed and circumscribed in and about a triangle, and the distance between the centres. I shall not now discuss the meaning of the other factor, or attempt to verify the formulæ which have been given by Fuss, Steiner and Richelot, for the case of a polygon of 4, 5, 6, 7, 8, 9, 12, and 16 sides. See Steiner, Crelle, t. II. [1827] p. 289, Jacobi, t. III. [1828] p. 376; Richelot, t. v. [1830] p. 250; and t. xxxviII. [1849] p. 353.

2 Stone Buildings, July 9, 1853. 